\title{
ENFOQUES Y OBJETIVOS HACIA UNA AUDITORÍA INTEGRADA
}

\author{
Dr. CARLOSA. EGÚSQUIZA PEREDA \\ CPC ALBERTO MONTES FARRO, CPC MIGUEL DÍAZ INCHICAQUI, CPC LEONCIO JAVIER \\ MELGAREJO, Lic. OLINDA EGÚSQUIZA PEREDA, CPC ELMER ZANABRIA HUISA, CPC RAÚL \\ MELLADO CAJIGAS, ECON. BONIFACIO LEON FERNÁNDEZ, MARÍA V. BARRIOS RAMOS, \\ CLAUDIA VALENCIA CHUNGA, JUAN A. CASTELLANO ZUNIIGA, DAVID SALCEDO QUINTANA
}

\section{RESUMEN}

El resultado de esta investigación nos permite señalar que los enfoques y objetivos hacia una auditoría integrada, debe efectuarse bajo la visión filosófica amplia y globalizada, así como teniendo en cuenta a los factores condicionantes incluyendo los riesgos de las empresas e instituciones, en sus distintas etapas de su vida económica. Es necesario que en el ámbito académico, las universidades del país incluyan en el currículo de estudios, las características y la naturaleza de la auditoría integrada. Específicamente la implantación de la enseñanza y aplicación de la auditoría integrada, que evalúa en forma integral a los negocios que operan en un contexto cambiante y global. Asimismo, se asegura la relevancia, confiabilidad y oportunidad de la información, así como las eficiencia de las operaciones, bien sea alargando la vida económica de los negocios, implementando la reorganización parcial o integral; o puedan también los usuarios del informe decidir vender la empresa, etc.

\section{INTRODUCCIÓN}

En materia de la auditoría tradicional la evolución indica que hasta la gran crisis económica y financiera de los Estados Unidos de Norteamérica del año de 1933 en que el balance o los estados financieros auditados se certificaban. Era un periodo en que muchas firmas de auditores y contadores públicos tuvieron que afrontar juicios y sufrir penas privativas de la libertad; por ello el Instituto Americano de Contadores Públicos Titulados, efectuó trabajos de investigación y recomendó a la profesión contable: The Generally Accepted Auditing Standards o las normas de auditoría generalmente aceptadas, que rigen hasta nuestros días, fundamentalmente para la ejecución de las auditorías contables y administrativas. En Canadá, los Estados Unidos de Norteamérica y Gran Bretaña, se vienen aplicando la auditoría operativa, de gestión o de las tres es (economía, eficiencia y eficacia); sobre todo en las entidades gubernamentales desde el año 1970; entre tanto, en el Perú se viene difundiendo y aplicando medianamente, a partir del año 1980, con énfasis en las empresas privadas.

La auditoría tradicional de cumplimiento administrativo o contable, relativo al POST MORTUM de las actividades económicas, financieras de las instituciones o empresas, que no aporta valor agregado, no es de aplicación óptima en los tiempos actuales de calidad, excelencia, modernidad y globalización. Por lo tanto, la auditoría no es sólo para cierta actividad humana, es decir para los negocios o actividades gubernamentales de ejecución presupuestal o de inversiones; también mediante el enfoque moderno de la auditoría, se aplica a todas las actividades y conocimientos de la humanidad.

En la Conferencia Interamericana de Contabilidad en Asunción-Paraguay, 
realizada en setiembre de 1993, se trató de la "Nueva frontera profesional" y de la "auditoría de la liberación", pensando, indudablemente ¿qué debe incluir y qué debe abarcar la auditoría moderna para el siglo XXI? Asimismo, es importante referirnos al Segundo Congreso Internacional de Auditoría Integral llevada a cabo en junio de 1995, en la ciudad de Buenos Aires, Argentina, en el que se dejaron las bases para que los investigadores y estudiosos en la materia se preocupen de efectuar trabajos en cada uno de los países involucrados, en tal sentido, con este trabajo se está dando cumplimiento el acuerdo anteriormente mencionado.

Por ello, para integrar y cohesionar esfuerzos y pensamientos, se ha efectuado el presente trabajo de investigación, para dar conocimientos hacia la necesidad de una auditoría integrada, a ser aplicada a las distintas actividades y vivencias del ser humano; que requiere involucrar a profesionales de distintas especialidades como economistas, contadores públicos, abogados, ingenieros, psicólogos, sociólogos, entre otros. En el presente trabajo se fijan los enfoques y objetivos hacia una auditoría integrada, abarcando con ello la auditoría contable y administrativa, auditoría operativa o de gestión, auditoría legal, auditoría de ingeniería, auditoría económica, auditoría ecológica y del medio ambiente, auditoría académica o de educación; porqué no, la auditoría de la equidad y de la ética entre otras; es decir bajo el enfoque integrador y globalizador.

En la actualidad, se viene efectuando la auditoría financiera o contable, regida por las normas de auditoría generalmente aceptadas, de la misma manera se vienen realizando las auditorías operativa y administrativas, tanto en las instituciones privadas y públicas. En cuanto a estas dos últimas, sobre todo en el Perú, sólo se viene efectuando una amplia- ción del examen del control administrativo contable, por falta de una información académica adecuada; por ello las empresas e instituciones a nivel gerencial y global carecen de una concepción teórica y práctica sobre la auditoría de gestión tendiente a la concepción de "ENFOQUES Y OBJETIVOS HACIA UNA AUDITORÍA INTEGRADA". En el Segundo Congreso de Auditoría Integral llevada a cabo en Buenos Aires en junio de 1995, además de fijarse las bases para que los investigadores y especialistas en la materia pudieran seguir investigando, especialmente en cuanto a las normas generales en materia de auditoría de gestión, se dieron las bases para que este tópico se conciba bajo el marco global de las instituciones y empresas, con la visión de calidad total, reingeniería y globalización.

\section{PLANTEAMIENTODELESTUDIO}

En el Perú y Latinoamérica se carece de un trabajo técnico científico encaminado a la evaluación o estudio de los problemas de decisión, operación y metas alcanzadas utilizando la auditoría de gestión institucional o empresarial con una visión global, abarcando desde el punto de vista de las distintas funciones de la empresa o institución (producción, comercialización, economía, finanzas, organización, administración, supervisión y control) Anexo $N^{\circ} 1$; y desde el punto de vista del "espacio y tiempo", o llamada también, vida económica de las empresas (periodos de inversión, de apogeo y de decadencia), ilustración gráfica $\mathrm{N}^{\circ} 02$; e incluyendo premisas básicas sobre la auditoría ecológico y medio ambiente; así como las auditorías: legal, de ingeniería, económica, académica, de equidad y ética, etc.

El planteamiento del presente estudio básicamente trata de bosquejar los nuevos enfoques y objetivos hacia una auditoría integrada a efectos de que sirva como 
instrumento de evaluación de problemas de decisión, operación y metas alcanzadas por la gerencia en un contexto globalizado; ya que los enfoques y objetivos de la auditoría tradicional han devenido en inoperantes por el desarrollo científico-tecnológico de las empresas e instituciones.

\section{DESCRIPCIÓN DEL TEMA}

El presente estudio referente al desarrollo de enfoques y objetivos, hacia una auditoría integrada, planteó para el logro de sus objetivos las siguientes hipótesis de trabajo:

1. En la actualidad las empresas o instituciones no vienen utilizando la auditoría integrada, debido a que no hay trabajos académicos ni técnico profesionales al respecto en el mercado.

2. Las universidades públicas y privadas no vienen impartiendo la auditoría integrada porque no la tienen incluida en sus currículos de estudios y las empresas o instituciones no están informadas sobre las ventajas y beneficios de trabajo técnico profesional de la magnitud y trascendencia de la auditoría integrada.

3. Los informes de auditoría tradicional no están dando resultados acorde de las necesidades de los accionistas, proveedores, clientes y gobierno, entre otros; que en vez de la descripción de hechos históricos, requieren propuestas concretas que permitan el crecimiento sostenido y competitivo de las organizaciones en general.

La metodología aplicada comprendió al tipo diseño experimental y procesamiento de información. En el diseño experimental, aparte de la descripción de los sistemas de gestión empresarial se explica el concepto de los enfoques desde el punto de vista filosófico, las características e indicadores de medida de aplicación en los diferentes sectores económicos.

El análisis documental consistió en la selección, procesamiento y evaluación de los informes de auditoría contable-administrativos, tanto el informe corto como la carta de control interno. También comprendió estudio y evaluación de los informes de auditoría operativa efectuada por contadores $\mathrm{y}$ auditores peruanos.

Siendo el ámbito de nuestra investigación a todas las instituciones empresariales que operan en el país, se diseño una encuesta y entrevista a directores de instituciones profesionales, privadas y públicas; así como a las principales empresas que operan en tres importantes ciudades (Lima, Arequipa y Huancayo), comprendiendo la aplicación a una muestra total de 44 instituciones y empresas. 


\section{CONCLUSIONES}

1. Los enfoques y objetivos hacia una auditoría integrada implica desarrollar una visión filosófica amplia tanto a las instituciones o empresas a auditarse en su quehacer operativo (interno y externo); formando metodologías y procedimientos más adecuados, acorde a los tiempos actuales de excelencia, valor agregado y costo beneficio.

2.La revisión de la bibliografía especializada sobre auditoría existente nos permite afirmar que en los países de Canadá, España, Argentina, México se están aplicando la auditoría integral, hacia la auditoría integrada; tópico que en nuestro medio es de escaso conocimiento y aplicación.

3. Las empresas e instituciones académicas que operan en el país vienen enfocando básicamente la auditoría tradicional a fin de cumplir con el marco normativo, olvidando el estudio, la evaluación y control de la vida económica de las instituciones y empresas, que permitan hacer frente a las exigencias de calidad y competitividad, $y$ en el contexto de una economía globalizada. Esta situación existente constituye aspecto limitante para el desempeño profesional del Contador Público con formación y experiencia sólo en la auditoría tradicional o financiera. Según la normatividad dada por la CONASEV sólo deben presentar estados financieros auditados, las empresas de mayor envergadura económico-financiera; y con este estudio se busca contribuir al mayor conocimiento. Sin embargo, para el cabal cumplimiento de los objetivos de una auditoría integral, existe la necesidad de participación de un equipo de profesionales multidisciplinarios (economistas, ingenieros, abogados, contadores, etcétera).

4. Los resultados de la aplicación de la encuesta y entrevista efectuada a una muestra de 44 instituciones y empresas representativas que operan en 3 principales ciudades del país, (Lima, Arequipa y Huancayo) confirmó como válida la hipótesis de trabajo planteados. Es decir los informes de auditoría de tipo tradicional son de aplicación ilimitada, puesto que los accionistas, proveedores, clientes, etc; más que del relato de hechos producidos, requieren propuestas concretas que tiendan hacia el crecimiento sostenido de las instituciones. Asimismo, las universidades que operan en el país, no vienen impartiendo ni incluyendo en sus currículums de estudios, la auditoría integrada.

\section{BIBLIOGRAFÍA}

1. XXIII CONGRESO INTERAMERICANO DE CONTADORES, San Juan - Puerto Rico, agosto,1999.

-Volumen I Trabajos Técnicos Interamericanos:

a) CAYETANO, Ángel V. /.SANTIESTEBAN HENTER, Jore H. / ALMEIDA ANDRADE, Guy/DEVACA PAVÓN, Angel.

"La auditoría integral, un enfoque integrador". -Volumen II Trabajos Técnicos Nacionales:

b) SÁNCHEZ HENQUEZ, Jorge

"La auditoría integral, precisiones sobre alcances y contenido".

c) FAX SANTÍBAÑEZ, Jorge Luis.

"La auditoría integral y el impacto ambiental".

2. La Auditoría que viene:

Conferencias Magistrales:

- "El nuevo concepto de auditoría global: Los otros auditores".

- "Hacia un modelo de auditoría interna de gestión".

- "La auditoría ambiental: Perspectiva del contador público y los otros auditores". - "Hacia una auditoría ética: Integridad y valores morales frente al fraude y corrupción". GONZÁLES MALAXECHEVARRÍA, Ángel Facultad de Ciencias Contables,Universidad 
Nacional Mayor de San Marcos, agosto, 1998.

\section{FRESCO, Juan Carlos}

1991." Desarrollo gerencial hacia la calidad total". Ediciones Macchi, Bogotá.

4. CONTADURÍA PÚBLICA DE LA NACIÓN Normas Internacionales de Contabilidad. enero, 1999. Lima.

\section{LATINOAMERICANA DE GERENCIA}

- Normas Internacionales de Contabilidad.

- Aplicaciones.

II. Seminario-Taller, enero,1999. Lima.

6.La evaluación del rendimiento operativo. ¿Cómo establecer y administrar una auditoría integral?

PRICE WATERHOUSE

\section{Revista OLACEFS}

Órgano de Difusión de la Organizaciones Latinoamericano y del Caribe de Entidades fiscalizadora Superiores:

Colombia: La auditoría integral, una herramienta eficiente para organizaciones fiscalizadoras. marzo, 1999.

8. Guía de Auditoría:

a) Norma de Auditoría ( SAS).

b) Testimonio (SSAE).

c) Servicios de Contabilidad y Revisión (SSACS).

d) Control de Calidad (SQCS).

Harcour Brace de España S.A.

Madrid, 1998.

9. Derecho y medio ambiente: Aproximaciones y estimativas.

Pontificia Universidad Católica del Perú Facultad de Derecho, Fondo Editorial, 1997. DE TRAZEGNIES, Fernando/FOX, Pierre / FIGALLOGuillermo y otros.

10. La responsabilidad de la contabilidad frente al medio ambiente.
Instituto Mexicano de Contadores Públicos, agosto,1998. México.

ANTRISTAN MOUNSERRAT, Patricia / ALVA

Ricardo/REZB.

11. Diccionario de Términos Contables:

Inglés - Español. Español - Inglés.

Instituto Mexicano de Contadores Públicos.

México, 1998.

12. ELIZARDO LÓPEZ, Arturo

1992. "La investigación contable: Significado y metodología". ECASA, México.

13. PINEDA, Pilar

1995. "La auditoría de la formación".

Ediciones Gestión 2,000 S.A.

Barcelona, España

14. R.K. MAUTZ

1991. "La filosofía de la auditoría".

Ediciones Contables y Administrativas.

15. SUÁREZ SUÁREZ, Andrés

1990. "La auditoría, un análisis conceptual y metodológico". Ediciones Mc. Graw Hill Printed in Spain.

16. TREVENET, Maurice

1992. "Auditoría de la cultura empresarial". Ediciones de Santos S.A., Madrid, España.

17. ABT, Clark C.

1981. "Auditoría social para la gerencia".

Editorial Diana, Mexico.

18. SLOOSE, Carlos A.

1994. "Auditoría un nuevo enfoque empresarial". Editorial Macchi, Buenos Aires.

19. DOUGLAS I. J./THOMAS A.J.

1987."Auditoría informática".Editorial Paraninfo, Madrid.

20. Auditoría computarizada: Control Interno AMERICAN INSTITUTE OF CERTIFIED PUBLIC ACCOUNTANTS 
1996. El control interno - Elementos de un sistema coordinado y su importancia para la gerencia y el auditor independiente.

Ediciones ECOE, EE.UU.

21. DRUCKER, Peter F.

Gerencia para el Futuro.

Grupo Editorial Norma

22. CHAVEZ ACKERMANN, Pascual 1999. Contabilidad: Una visión al futuro EDYP. Lima, Penú.

23. FEDERACIÓN DE COLEGIO DE CONTADORES PÚBLICOS DEL PERÚ

1997. XXII Conferencia Interamericana de Contabilidad.

24. Trabajos Técnicos Interamericanos Area de Auditoría.

Colegio de Contadores Públicos autorizados del Puerto del Perú.

XXII Conferencia Interamericana de Contabilidad, 1999.

25. Memoria del Segundo Congreso Internacional de Auditoría Integral.

La auditoría integral ante el desafío de la globalización. SIGEN. Buenos Aires, junio de 1995.

26. M. IVANCEVICH, John - Peter Lorenzi Steven J. SKINNER y Philip CROSBY 1996. "Gestión, calidad y competitividad" IRVIN.

27. HAMMER, Michael \& CHAMPY, James 1996. Reingeniería. Grupo Editorial Norma.

28. SLOSSE, C.A.

1994. "Auditoría: Un nuevo enfoque empresarial". Macchi Grupo Editor. 
LA EMPRESA DESDE EL PUNTO DE VISTA DE LAS OPERACIONES/TRANSACCIONES

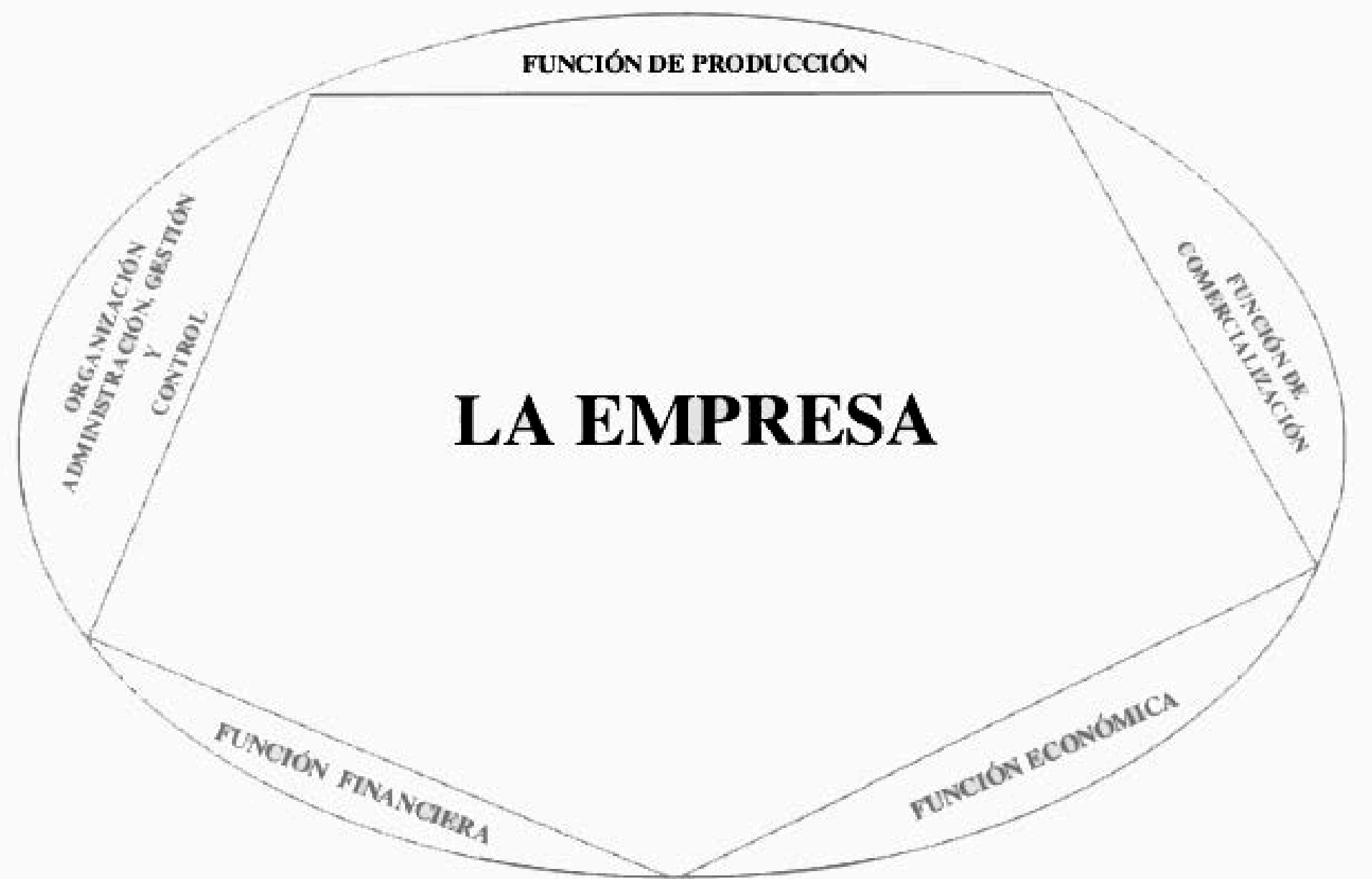

Bajo este enfoque, se puede hacer Auditoría a una de las funciones o a todas, en busqueda de la venta o reorganización 


\section{LA EMPRESA VISTA A TRAVÉS DEL ESPACIO Y TIEMPO CURVA DE LA VIDA ECONÓMICA}

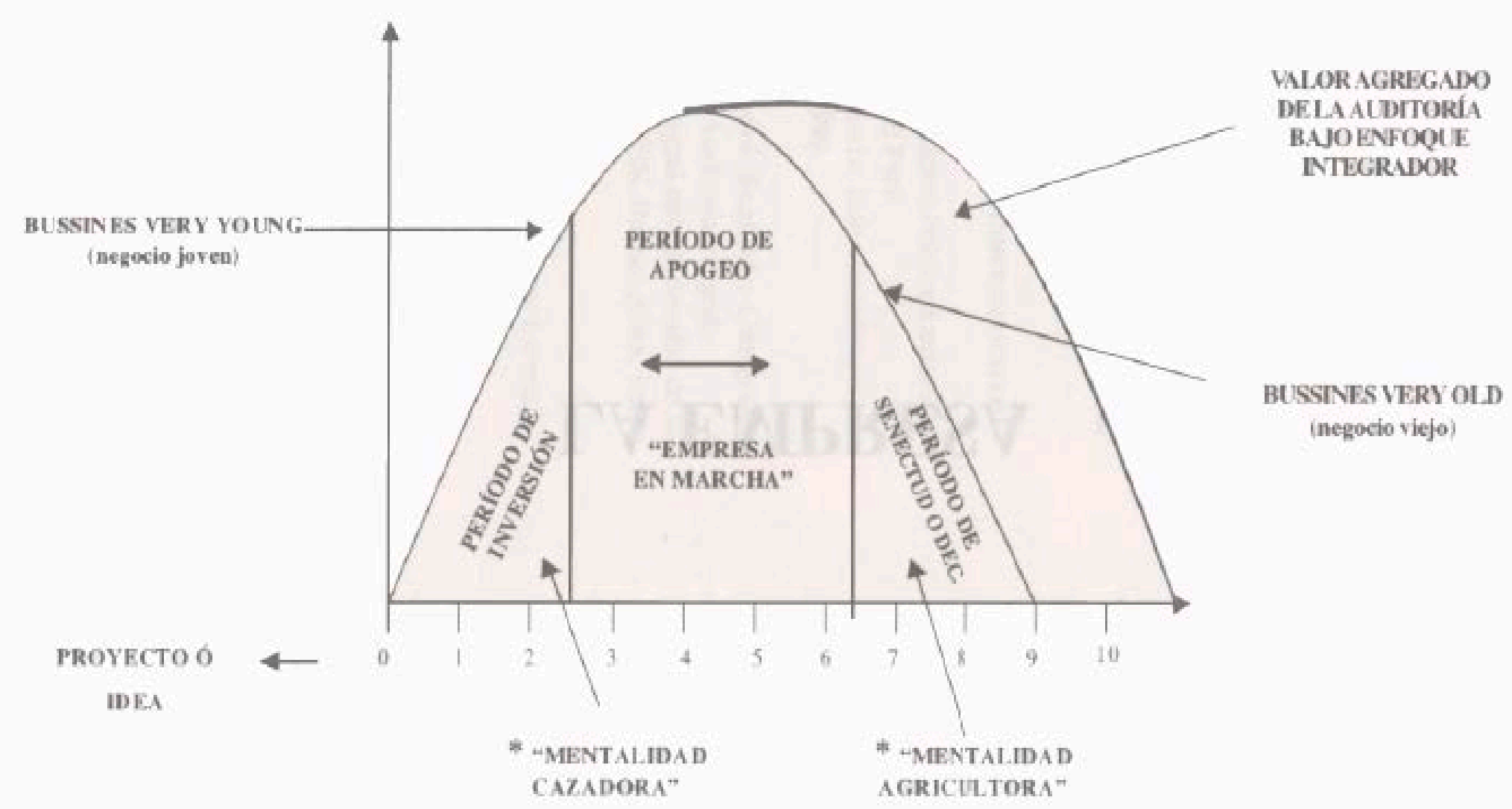

